\title{
Light-Emitting Diodes with Cu-Doped Colloidal Quantum Wells: From Ultrapure Green, Tunable Dual-Emission to White Light
}

\author{
Baiquan Liu, Manoj Sharma, Junhong Yu, Sushant Shendre, Chathuranga Hettiarachchi, \\ Ashma Sharma, Aydan Yeltik, Lin Wang, Handong Sun, Cuong Dang, \\ and Hilmi Volkan Demir**
}

Copper-doped colloidal quantum wells (Cu-CQWs) are considered a new class of optoelectronic materials. To date, the electroluminescence (EL) property of Cu-CQWs has not been revealed. Additionally, it is desirable to achieve ultrapure green, tunable dual-emission and white light to satisfy the various requirement of display and lighting applications. Herein, light-emitting diodes (LEDs) based on colloidal $\mathrm{Cu}-\mathrm{CQW}$ are demonstrated. For the $0 \% \mathrm{Cu}$-doped concentration, the LED exhibits Commission Internationale de L'Eclairage 1931 coordinates of $(0.103,0.797)$ with a narrow EL full-wavelength at half-maximum of $12 \mathrm{~nm}$. For the $0.5 \%$ Cu-doped concentration, a dual-emission LED is realized. Remarkably, the dual emission can be tuned by manipulating the device engineering. Furthermore, at a high doping concentration of $2.4 \%$, a white LED based on CQWs is developed. With the management of doping concentrations, the color tuning (green, dual-emission to white) is shown. The findings not only show that LEDs with CQWs can exhibit polychromatic emission but also unlock a new direction to develop LEDs by exploiting 2D impurity-doped CQWs that can be further extended to the application of other impurities (e.g., Mn, Ag).

Colloidal quantum wells (CQWs), also referred to as semiconductor nanoplatelets (NPLs) or atomically flat nanocrystals, have been revealed as a new family of 2D nanocrystals in the past few years. ${ }^{[1-4]}$ Unlike colloidal quantum dots (CQDs), CQWs exhibit many unique thickness-dependent optical properties due to the strong quantum confinement only in the vertical direction. These properties include a narrow emission spectrum, suppressed inhomogenous emission broadening, giant oscillator strength, an ultrashort radiative fluorescence lifetime, and extremely large linear/nonlinear absorption crosssections. ${ }^{[5-8]}$ Due to these excellent characteristics, CQWs have been actively studied for different optoelectronic applications including lasers, photovoltaics, and lightemitting diodes (LEDs). ${ }^{[9-11]}$

As a new type of nanocrystal LEDs, CQW-LEDs have great potential for displays and lighting thanks to their merits such as outstanding color purity, low power consumption, high efficiency, and flexibility. ${ }^{[12-14]}$ The first CQW-LED was emerged in 2014, ${ }^{[15]}$ which was two decades later than the first CQD-LED. [16]

Thus, understanding why the performance of CQW-LEDs lags behind that of CQD-LEDs is not difficult. However, by virtue of the knowledge of other types of LEDs (e.g., CQD-LEDs and organic LEDs) and the increased understanding of the photophysics of CQWs, ${ }^{[17-20]}$ the development of CQW-LEDs is accelerating. To date, various architectures
Dr. B. Liu, Dr. M. Sharma, J. Yu, S. Shendre, A. Sharma, Prof. C. Dang, Prof. H. V. Demir

Luminous! Centre of Excellence for Semiconductor Lighting and Displays School of Electrical and Electronic Engineering and School of Physical and Mathematical Sciences

Nanyang Technological University

Singapore 639798, Singapore

E-mail: hvdemir@ntu.edu.sg

C. Hettiarachchi, Prof. C. Dang

Centre for OptoElectronics and Biophotonics (COEB)

School of Electrical and Electronic Engineering

The Photonics Institute (TPI)

Nanyang Technological University

50 Nanyang Avenue, Singapore 639798, Singapore

The ORCID identification number(s) for the author(s) of this article can be found under https://doi.org/10.1002/smll.201901983.
Dr. A. Yeltik, Prof. H. V. Demir

Department of Electrical and Electronics Engineering

Department of Physics

UNAM-Institute of Materials Science and Nanotechnology

Bilkent University

Ankara 06800, Turkey

Dr. L. Wang, Prof. H. Sun

Division of Physics and Applied Physics

School of Physical and Mathematical Sciences

Nanyang Technological University

Singapore 637371, Singapore

DOI: 10.1002/smll.201901983 
(e.g., core-only, core/shell, and core/crown) based CQWs have been used for LEDs. For example, Fan et al. fabricated an LED with $\mathrm{CdSe}_{1-x} \mathrm{~S}_{x}$ alloyed core-only CQWs, achieving a maximum luminance of $\approx 90 \mathrm{~cd} \mathrm{~m}^{-2}$, which is currently the best value for inverted green CQW-LEDs. ${ }^{[21]}$ Furthermore, we previously reported a red $\mathrm{LED}$ with $\mathrm{CdSe} / \mathrm{CdSe}{ }_{0.8} \mathrm{Te}_{0.2}$ core/ crown type II CQWs, obtaining a maximum external quantum efficiency (EQE) of 3.57\%.[22] More recently, Giovanella et al. reported a deep-red LED by adopting CdSe/CdZnS core/shell CQWs, yielding an EQE of $8.39 \%$ and a maximum luminance of $\approx 100 \mathrm{~cd} \mathrm{~m}^{-2}$. [23] Therefore, CQW-LEDs have great potential to show high performance. However, despite the tendency of CQW-LEDs to furnish saturated red colors, the reported green color is not sufficiently pure. In addition, the previous CQWLEDs only exhibited monochromatic electroluminescence (EL), with no chance of dual emission. Furthermore, white emission based on CQWs has not been documented. Hence, a large gap between CQW-LEDs and other types of LEDs still exists.

Since the human eye is more sensitive to green than to blue/red colors, meeting the demand of the International Telecommunication Union Recommendation BT 2020 (Rec. 2020) standard for new-generation ultrahigh definition TVs is challenging for green LEDs. ${ }^{[2]}$ To loosen this bottleneck, many efforts have been made to enlarge the color gamut which is defined by the corresponding three color primaries (e.g., the Commission Internationale de L'Eclairage (CIE) 1931 color coordinates of $(0.170,0.797)$ for the Rec. 2020 standard green emitter). ${ }^{[2]}$ For instance, Kumar et al. demonstrated a green LED with 2D formamidinium lead bromide hybrid perovskites, achieving a color gamut covering $96.8 \%$ of the Rec. 2020 standard in the CIE 1931 color space, which is the "greenest" LED reported thus far. ${ }^{[25]}$ Using supercrystal inorganic perovskites, Tong et al. also reported an LED exhibiting a color close to the Rec. 2020 pure green. ${ }^{[26]}$ On the other hand, dual-emission colloidal semiconductor nanocrystals are of interest for applications in multimodal imaging, sensing, lighting, and integrated photonics. ${ }^{[27-29]}$ However, the best EQE of single-nanocrystal LEDs with dual emission cannot exceed $0.100 \% .{ }^{[30]}$ In addition, no report that the dual emission of single-nanocrystal LEDs can be tuned by manipulating the device engineering exists. Furthermore, although white emission is desirable for lighting and high-quality displays, the study of white LEDs (WLEDs) based on CQWs remains unexplored. Therefore, a question arises: is it possible for CQW-LEDs to produce ultrapure green, tunable dual-emission and white EL?

Impurity doping is a widespread method to afford semiconductor nanocrystals possessing new optical, electronic, and magnetic characteristics. ${ }^{[31]}$ Over the last decade, many endeavors have been undertaken in the development of $\mathrm{Cu}$ doped $0 \mathrm{D}$ and $1 \mathrm{D}$ nanocrystals. ${ }^{[32-34]}$ Recently, we have reported Cu-doped 2D CQWs and showed that they are promising for solar light harvesting technologies. ${ }^{[35]}$ However, the effect of Cu-doped CQWs in LEDs has not yet been elucidated. In fact, no LED based on impurity-doped CQWs has been reported thus far.

In this paper, for the first time, LEDs based on Cu-doped CQWs have been developed. Specifically, by controlling the $\mathrm{Cu}$ concentration in CdSe CQWs, the Cu-doping effect in LEDs has been revealed. For the $0 \% \mathrm{Cu}$-doped concentration, the
LED exhibits the CIE 1931 coordinates of (0.103, 0.797). Significantly, the full-wavelength at half-maximum (FWHM) of the EL emission is as narrow as $12 \mathrm{~nm}$. For a $0.5 \% \mathrm{Cu}$-doped concentration, the LED possesses dual emission with an EQE of $0.146 \%$. To the best of our knowledge, i) this LED is the first CQW-LED possessing dual emission, ii) the efficiency is the best for single-nanocrystal LEDs with dual emission, iii) the approach is a new one to vastly enhance the performance of CQW-LEDs via impurity doping (i.e., realizing nine times higher EQE than the 0\% concentration-based device). Impressively, this dual emission can be tuned by manipulating the device engineering, which is an unexplored emission mechanism for single-nanocrystal LEDs. Moreover, at a high Cudoped concentration of $2.4 \%$, a new WLED based on CQWs has been demonstrated.

Cu-doped CdSe CQWs were synthesized by a high-temperature nucleation doping method recently reported by our group. ${ }^{[35]}$ Further details of the syntheses can be found in the Supporting Information. The optical properties of these undoped and Cu-doped CQWs were characterized via UV-vis absorption and photoluminescence (PL) spectroscopy. Figure 1a shows the steady-state UV-vis absorption and PL emission spectra of $0 \%, 0.5 \%$, and $2.4 \% \mathrm{Cu}$-doped CdSe CQWs. The atomic ratio of $\mathrm{Cu}$ dopant ions with respect to total cations $(\mathrm{Cu}+\mathrm{Cd})$ was measured by inductively coupled plasma mass spectrometry (ICP-MS). The sharp excitonic features at $\approx 480$ and $\approx 512 \mathrm{~nm}$ observed in the absorption spectra of the CQWs are associated with the electron-light hole and electronheavy hole transitions, respectively. With successful Cu-doping in $4 \mathrm{ML}$ CdSe CQWs, a broad and Stokes-shifted emission peak $(\approx 700 \mathrm{~nm}$ ) appears along with the usual excitonic bandedge emission peak ( $\approx 515 \mathrm{~nm}) \cdot{ }^{[35]}$ With an increase in the $\mathrm{Cu}$ doping value, the contribution of the dopant emission increases with respect to the total integrated emission (excitonic $+\mathrm{Cu}$ dopant emission). For $0.5 \%$ Cu-doped CdSe CQWs, decay traces at the excitonic $(515 \mathrm{~nm})$ and dopant $(700 \mathrm{~nm})$ emissions were obtained using time-resolved fluorescence spectroscopy. Clearly, the dopant emission shows a long excited state lifetime compared to the order-of-magnitude-faster excitonic lifetime (Figure 1b, Tables S1 and S2, Supporting Information). Furthermore, the absence of a fast lifetime decay channel $(\approx 10-20 \mathrm{~ns})$ for the dopant emission clearly indicates efficient dopant emission in these CQWs (Table S2, Supporting Information). The origin of this highly efficient and Stokes-shifted emission has been discussed in detail in our previous work. ${ }^{[35]}$ The Cu-doped CdSe CQWs exhibit a roughly rectangular shape, as seen from the high-angle annular dark-field scanning transmission electron microscopy (HAADF-STEM) image shown in Figure 1c. The average dimensions measured from the STEM image are $32.0 \times 13.5 \mathrm{~nm}^{2}$. To estimate the $\mathrm{Cu}$ doping concentration, by using STEM-based energy-dispersive X-ray spectroscopy (EDX), we estimated the $\mathrm{Cu}$ atomic percentage to be $1.1 \%$ with respect to cadmium levels for a lightly doped sample, which shows a higher value as compared with ICP-MS results discussed above (Figure 1d). Therefore, in the whole study we show $\mathrm{Cu}$ doping levels by ICP:MS measurements unless otherwise stated.

The device architecture of the CQW-LEDs is indium tin oxide (ITO)/zinc oxide (ZnO)/CQWs/4,4- $N$, $N$-dicarbazolebiphenyl $(\mathrm{CBP}) / \mathrm{MoO}_{3} / \mathrm{Al}$, where ITO serves as the cathode, $\mathrm{ZnO}$ as the 

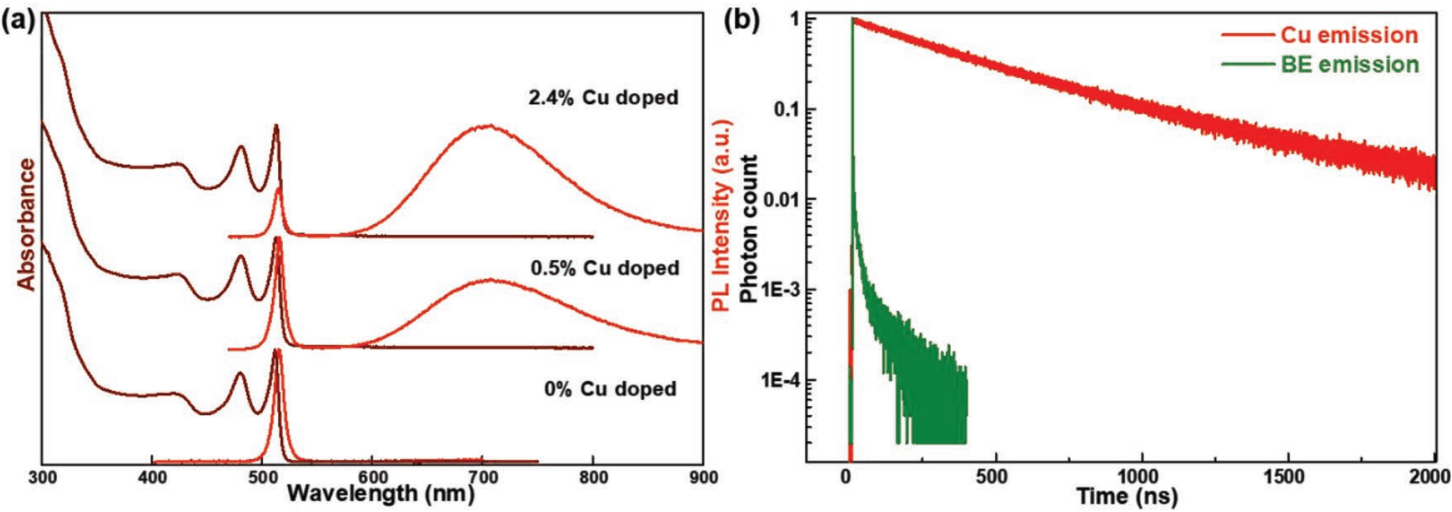

(c)
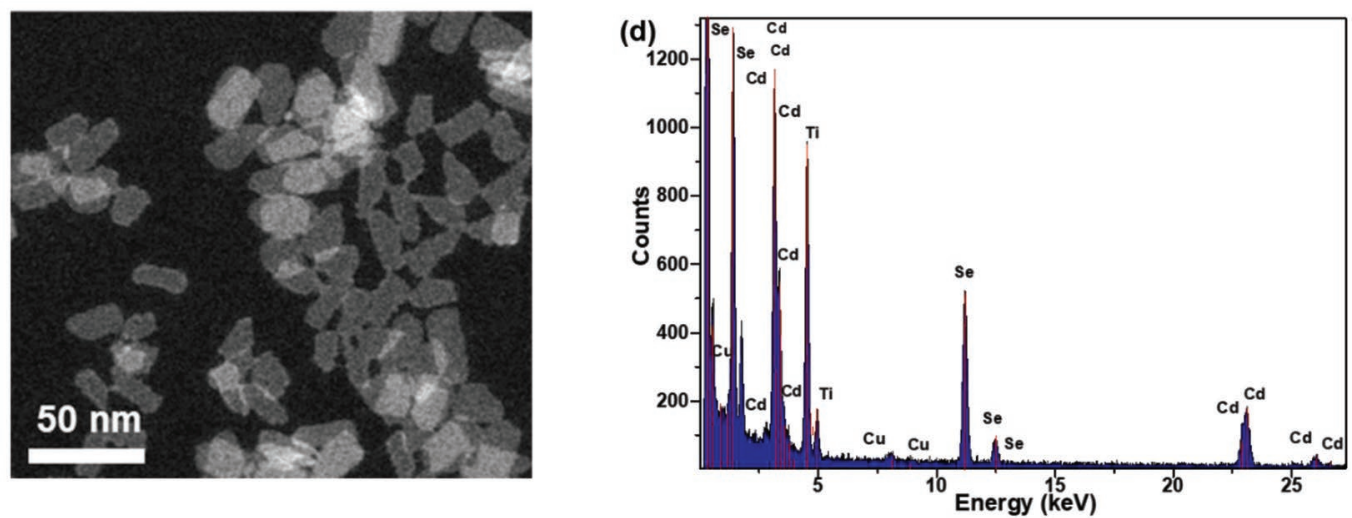

Figure 1. a) Room-temperature absorption and PL emission spectra of CQWs with different Cu concentrations in hexane solution. b) Time-resolved fluorescence decay traces of $0.5 \%$ Cu-doped CQWs at the band-edge (BE) and dopant emissions. c) HAADF-STEM image of the Cu-doped CdSe CQWs with a $0.5 \%$ Cu concentration. d) STEM-EDX spectra of $0.5 \%$ Cu-doped CQWs.

electron transport layer, the CQWs as the emitting layer (EML), CBP as the hole transport layer (HTL), $\mathrm{MoO}_{3}$ as the hole injection layer, and $\mathrm{Al}$ as the anode, as shown in Figure 2a. Given the high electron mobility of $\mathrm{ZnO}\left(1.8 \times 10^{-3} \mathrm{~cm}^{2} \mathrm{~V}^{-1} \mathrm{~s}^{-1}\right)^{[18]}$ and high hole mobility of CBP $\left(1.0 \times 10^{-3} \mathrm{~cm}^{2} \mathrm{~V}^{-1} \mathrm{~s}^{-1}\right)^{[22]}$ together with the fact that the barrier between the charge transport layers and CQWs is not high (Figure 2b), both electrons and holes can efficiently arrive at the EML. Therefore, with this configuration, charges can be effectively transported to the $\mathrm{CQWs}$ to generate excitons, which is beneficial for exploring the effect of Cu-doped CQWs in LEDs.

To examine the electrical characteristics, CdSe CQWs with a $0 \% \mathrm{Cu}$-doped concentration were first used as the emitter for LEDs (Device A). A cross-sectional scanning electron microscope image of Device A is depicted in Figure S1 (Supporting Information). As shown in Figure $2 \mathrm{c}$ and Table 1, the maximum EQE and current efficiency (CE) of Device A are $0.016 \%$ and $0.055 \mathrm{~cd} \mathrm{~A}^{-1}$, respectively. In addition, the maximum power efficiency (PE) is $0.040 \mathrm{~lm} \mathrm{~W}^{-1}$ (Figure S2a, Supporting Information). Notably, these efficiencies are the highest reported for inverted green CQW-LEDs. Additionally, the driving voltage is very low. For example, the turn-on voltage (the voltage when the luminance can be detected, $\geq 0.01 \mathrm{~cd} \mathrm{~m}^{-2}$ ) is approximately $2.1 \mathrm{~V}$, which is among the lowest values reported for nanocrystalbased LEDs. This voltage is lower than the bandgap of CdSe CQWs $(2.47 \mathrm{eV})$, which can be called as sub-bandgap voltage. ${ }^{[21]}$
Such sub-bandgap voltage has also been observed by other groups, which can be attributed to the efficient charge injection at low bias (i.e., the use of effective $\mathrm{ZnO}$ and $\mathrm{CBP}$ charge transport layers ensure the charge injection in our LEDs). ${ }^{[36]}$ The voltage is about $3.1 \mathrm{~V}$ when the luminance is $1 \mathrm{~cd} \mathrm{~m}^{-2}$. Remarkably, the maximum luminance is $210 \mathrm{~cd} \mathrm{~m}^{-2}$ (Table S3, Supporting Information), which is $>2$ times higher than that of the best inverted green CQW-LED. ${ }^{[21]}$ As shown in Figure 2e,f, Device A exhibits a green EL emission with the CIE 1931 coordinates of $(0.103,0.797)$. As a result, the color gamut covers 104\% of the Rec. 2020 standard in the CIE 1931 color space, demonstrating the purest green emission ever reported. ${ }^{[25,26]}$ In terms of the National Television System Committee standard, the color gamut is as high as $123 \%$ in the CIE 1931 color space. Significantly, the FWHM of the EL emission is only $12 \mathrm{~nm}$. Such extremely narrow emission may be attributed to the fact that CdSe CQWs possesses strong quantum confinement in 1D due to their fixed vertical thickness at atomic levels. ${ }^{[1]}$ By comparison with previous reports of the narrowest EL FWHMs for different types of LEDs (e.g., $16 \mathrm{~nm}$ for $\mathrm{CsPbBr}_{3}$ green perovskite LEDs, ${ }^{[37]} 14 \mathrm{~nm}$ for $\mathrm{PEA}_{2} \mathrm{PbBr}_{4}$ LEDs, ${ }^{[38]}$ and $15.3 \mathrm{~nm}$ for 3D perovskite thin film LEDs ${ }^{[39]}$ ), CdSe-based CQW-LEDs have been demonstrated here to show superior color purity.

By doping $0.5 \% \mathrm{Cu}$ into $\mathrm{CdSe}$ CQWs, an LED based on this emitter was developed (Device B), where the architecture of Device B is similar to that of Device A except for the EML. 
(a)

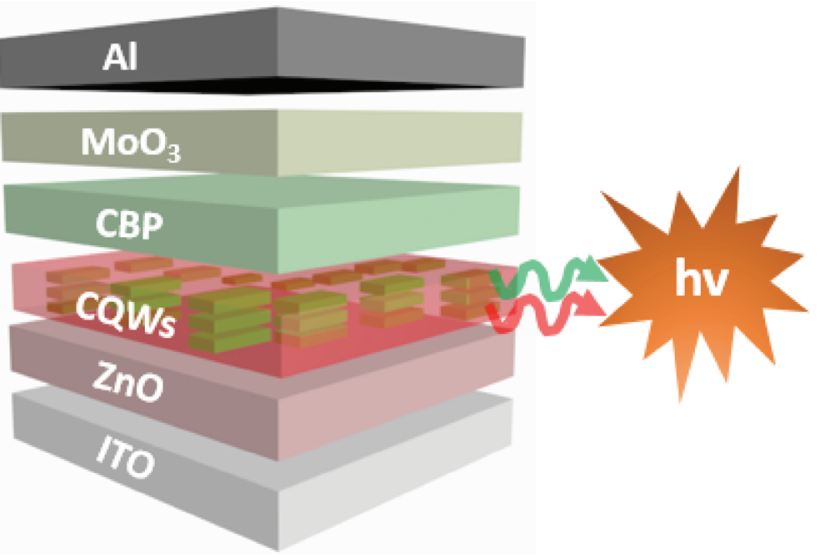

(b)

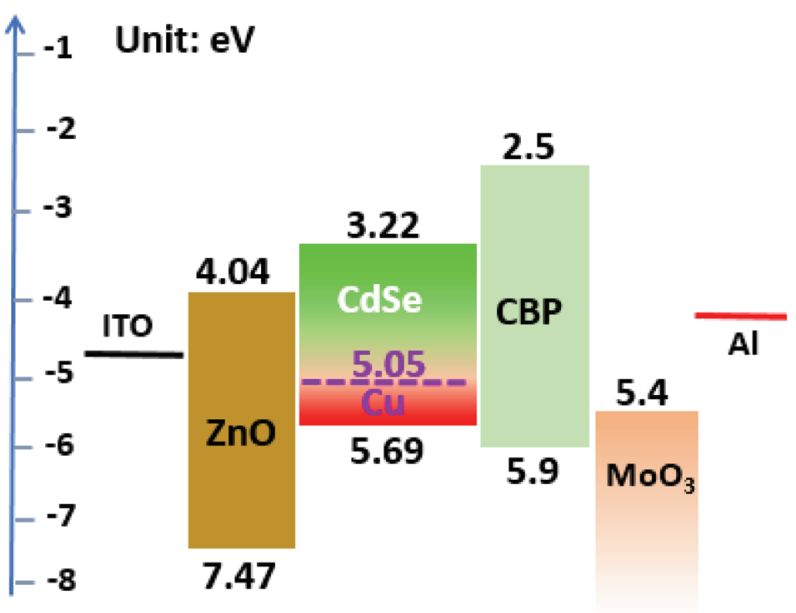

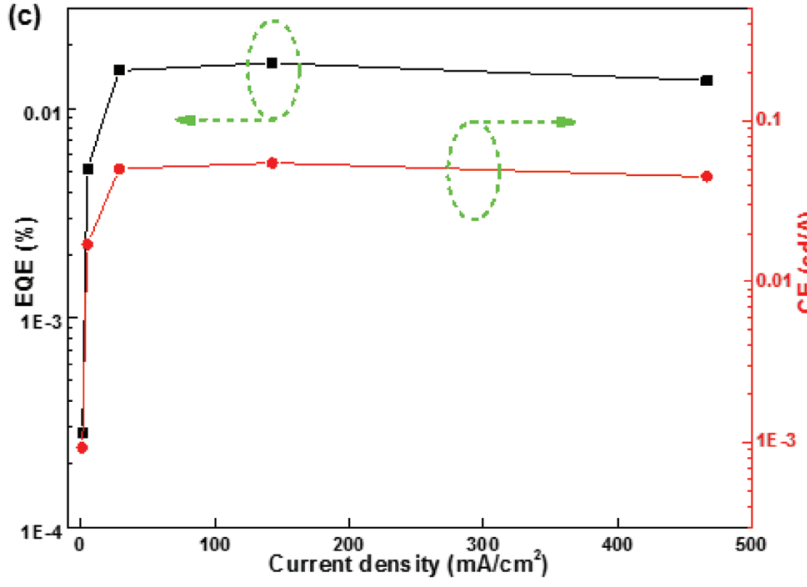
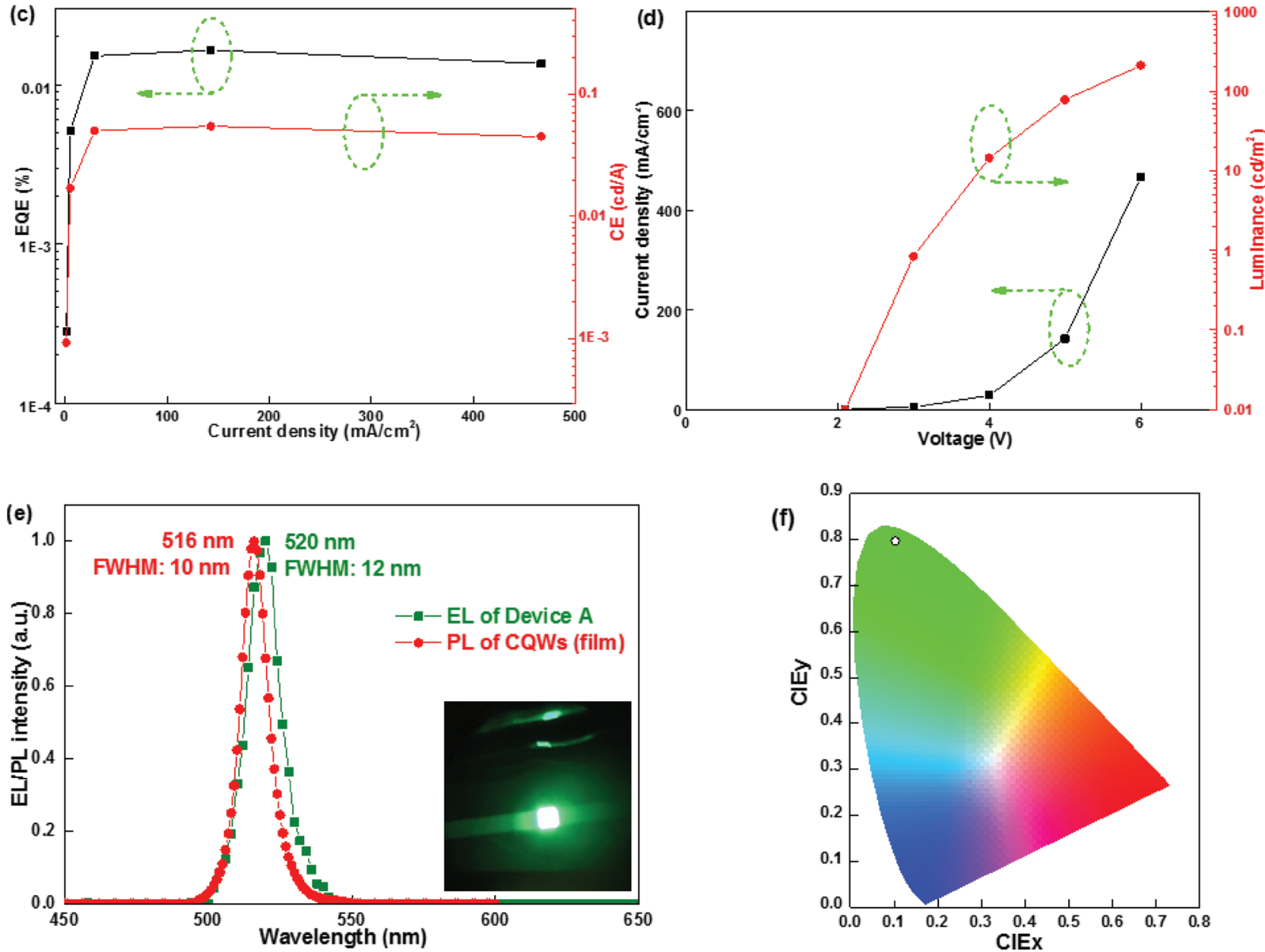

Figure 2. a) Schematic architecture of CQW-LEDs. b) The proposed energy levels of CQW-LEDs. The unit of energy is eV. The values are taken from the literature. ${ }^{[18,22,40-42]}$ c) EQE and CE of Device A. d) Current density and luminance of Device A. e) The EL spectrum of Device A and the PL spectrum of CdSe CQWs with a 0\% Cu-doped concentration film. Compared with the PL spectrum of the film, with an emission of $516 \mathrm{~nm}$ and an FWHM of $10 \mathrm{~nm}$, the EL spectrum of Device A is slightly redshifted $(520 \mathrm{~nm})$, and the EL FWHM is relatively widened $(12 \mathrm{~nm})$. Inset: a photograph of Device A under bias. f) The CIE 1931 coordinates of Device A in the color space.

As shown in Figure 3a, the maximum EQE of Device $B$ is $0.146 \%$, which is nine times greater than that of Device A and is the highest reported value for single-nanocrystal LEDs with dual emission. ${ }^{[30]}$ Previously, Chen et al. achieved a twofold increase in the EQE of CQW-LEDs by exchanging the long ligands of CQWs with short ones, ${ }^{[15]}$ while Giovanella et al. 
Table 1. Summary of LED performances.

\begin{tabular}{|c|c|c|c|c|c|c|}
\hline Device & $E M L^{a)}$ & $\begin{array}{c}\mathrm{EQE}_{\max }^{\mathrm{b})} \\
{[\%]}\end{array}$ & $\begin{array}{l}\left(E_{\max }{ }^{c)}\right. \\
{\left[c d A^{-1}\right]}\end{array}$ & $\begin{array}{l}\mathrm{PE}_{\max }{ }^{\mathrm{d})} \\
{\left[\mathrm{Im} \mathrm{W}^{-1}\right]}\end{array}$ & $\begin{array}{l}V_{\text {on }}{ }^{e)} \\
{[\mathrm{V}]}\end{array}$ & $\begin{array}{c}\left.L_{\max }^{f}\right) \\
{\left[\mathrm{cd} \mathrm{m}^{-2}\right]}\end{array}$ \\
\hline A & $0 \%$ Cu-doped CQWs & 0.016 & 0.055 & 0.040 & 2.1 & 210 \\
\hline B & $\begin{array}{c}0.5 \% \text { Cu-doped } \\
\text { CQWs }\end{array}$ & 0.146 & 0.282 & 0.179 & 2.4 & 1153 \\
\hline $\mathrm{E}$ & $\begin{array}{c}\text { ZnCdS/ZnS CQDs: } \\
2.4 \% \text { Cu-doped } \\
\text { CQWs }\end{array}$ & 0.029 & 0.025 & 0.020 & 2.3 & 148 \\
\hline
\end{tabular}

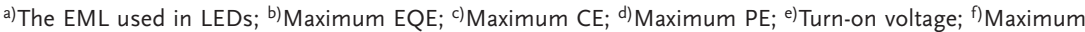
luminance.

obtained a 1.5-fold increase in EQE by exposing CQW-LEDs to air. ${ }^{[23]}$ Here, we have accomplished a ninefold increase in EQE by doping impurities into CQWs, unlocking a new strategy to vastly enhance the efficiency of CQW-LEDs. Since the error of EQE can be analyzed by the reproducibility, ${ }^{[18]}$ we have measured the reproducibility of Devices A and B. As shown by the histograms of maximum EQE taken from 18 devices, the reproducibility of devices is good (Figure S3, Supporting Information). Additionally, the maximum CE, PE, and luminance of Device B are $0.282 \mathrm{~cd} \mathrm{~A}^{-1}$ (Figure 3a), $0.179 \mathrm{~lm} \mathrm{~W}^{-1}$ (Figure S2b, Supporting Information), and $1153 \mathrm{~cd} \mathrm{~m}^{-2}$ (Figure 3b), respectively, which are much higher than those of Device A. The maximum current density of Device B is $1536 \mathrm{~mA} \mathrm{~cm}^{-2}(9.8 \mathrm{~V})$, which is higher than that of Device A $\left(466 \mathrm{~mA} \mathrm{~cm}^{-2}, 6.0 \mathrm{~V}\right)$. Hence, the doped LEDs exhibit a better stability than undoped ones since high voltage usually causes high working temperature (Joule heating). ${ }^{[43]}$ Furthermore, because the stability can be judged from the half-lifetime of devices, ${ }^{[18]}$ we have measured the lifetime at an initial luminance of $210 \mathrm{~cd} \mathrm{~m}^{-2}$, where the voltages are 6.0 and $\approx 6.3 \mathrm{~V}$ for Devices $\mathrm{A}$ and $\mathrm{B}$, respectively. As expected, the lifetime of Device B is $120 \mathrm{~s}$, which is much better than that of Device A (20 s). However, the high temperature is detrimental to the efficiency of LEDs, leading to an efficiency droop. In addition, the charge balance may be weaker at high voltage, which is another factor for the efficiency droop. ${ }^{[44]}$ Given that using the terminology of turn-on is dependent on sensitivity or dynamic range of instruments, the utilization of operating voltage requirement to achieve a specific current density is also a good practice. ${ }^{[45]}$ For example, the current density of $1 \mathrm{~mA} \mathrm{~cm}{ }^{-2}$ is achieved at 2.1 and $\approx 2.9 \mathrm{~V}$ for Devices $\mathrm{A}$ and $\mathrm{B}$, respectively. This fact may suggest that the current density of devices is lowered due to the effect of $\mathrm{Cu}$ dopant. As shown in Figure 3c, Device B can exhibit dual EL emissions at various voltages, where the peaks at $\approx 516$ and $\approx 692 \mathrm{~nm}$ originate from the host $\mathrm{CdSe}$ and $\mathrm{Cu}^{+}$ions, respectively.

The fact that the efficiency of CQW-LEDs can be vastly enhanced via impurity doping is an unprecedented phenomenon. To understand this phenomenon, the emission mechanism of Device B was revealed. The PL quantum yield (PLQY) of the $0.9 \%$ Cu-doped CQWs used for LEDs $(48.5 \%)$ is higher than that of the $0 \% \mathrm{Cu}$-doped CQWs utilized for LEDs (10\%), which can be attributed to the fact that the $\mathrm{Cu}$ doping results in an additional dominant radiative decay channel that is induced by mid-gap $\mathrm{Cu}(\mathrm{I})$ states. ${ }^{[35]}$ Importantly, the PLQYs of Cu-doped CdSe CQWs that are obtained after one cleaning of the synthesized samples are quite high $(\approx 80 \%)$. However, the doped CQWs used for our EL devices are typically cleaned three times with ethanol to remove excess ligand, which in turn helps to form better films in devices. The doped CQWs maintain fairly high PLQYs $(\approx 40-50 \%)$ even after excessive cleaning. Therefore, since the EQE is in direct proportion to the PLQY of the emitter, ${ }^{[22]}$ the efficiency of Device B is greatly enhanced. In addition, as shown in Figure $3 \mathrm{~d}$, the $\mathrm{Cu}$ energy level $(5.05 \mathrm{eV})$ is higher than the valance band maximum (VBM) of CdSe $(5.69 \mathrm{eV})$, indicating that the holes transported from CBP are more easily trapped by $\mathrm{Cu}$ under a low electrical field. ${ }^{[4]}$ Then, the holes meet the electrons transported from $\mathrm{ZnO}$ in the conduction band minimum (CBM) to generate excitons that recombine to produce the $\mathrm{Cu}^{+}$ emission. With increasing electrical field, holes trapped by $\mathrm{Cu}$ can be saturated, leading to relatively more holes transported from CBP being injected into the VBM of CdSe after saturation at the dopant site. These analyses can be confirmed by the EL spectra of Device B, as the $\mathrm{Cu}^{+}$emission is lowered with increasing voltage (Figure $3 \mathrm{c}$ ). To further verify the trapping effect of $\mathrm{Cu}$, the current densities of Devices A and B were compared (Figure S4, Supporting Information). As expected, the current density of Device B is lower than that of Device A, which indicates the trapping effect. ${ }^{[44]}$ From the current density-voltage characteristics as shown in Figure S4 (Supporting Information), we can estimate that the slope of Device B is nearly 0.5 times that of Device A. This implies that the ideality factor for the Device B is nearly twice that of Device A which suggests that Cu-doping acts as an impurity defect site. ${ }^{[46]}$

Therefore, two decay channels for exciton recombination exist in Device B (i.e., the excitons are recombined from the electrons of the CdSe CBM and the holes of the $\mathrm{Cu}$ level to produce $\mathrm{Cu}^{+}$emission or the excitons are recombined from the electrons of the CdSe CBM and the holes of the CdSe VBM to produce CdSe emission), which guarantee green/red dual emission, as shown in Figure 3c. Although holes are favored to be harvested by $\mathrm{Cu}^{+}$at low electrical fields, exciton recombination in $\mathrm{CdSe}$ and $\mathrm{Cu}^{+}$occurs simultaneously, which can be confirmed by the EL spectrum at the turn-on voltage. As shown in Figure S5 (Supporting Information), both CdSe and $\mathrm{Cu}^{+}$emissions can be distinguished at $2.1 \mathrm{~V}$. In the case of solution-processed LEDs, the surface roughness of the active layer plays an important role in the device performance. By using the atomic force microscopy, the root mean square roughness for the undoped CQW film is $4.02 \mathrm{~nm}$ (Figure S6, Supporting Information), which is higher than that of $\mathrm{Cu}$ doped film $(3.89 \mathrm{~nm})$. These values are consistent with previous papers (e.g., $3.65 \mathrm{~nm}$ for Fan's CQW films ${ }^{[21]}$ ). Hence, the improved morphology is another reason for the enhanced EQE of Device $B,{ }^{[47]}$ apart from the better PLQY of emitters and the simultaneous exciton recombination at the CdSe band edge and $\mathrm{Cu}$ dopant.

Previously, the dual emission of LEDs can be tuned via material design (i.e., adjusting the material compositions of emitters), which are easily understandable. ${ }^{[5,15,21]}$ For the case 

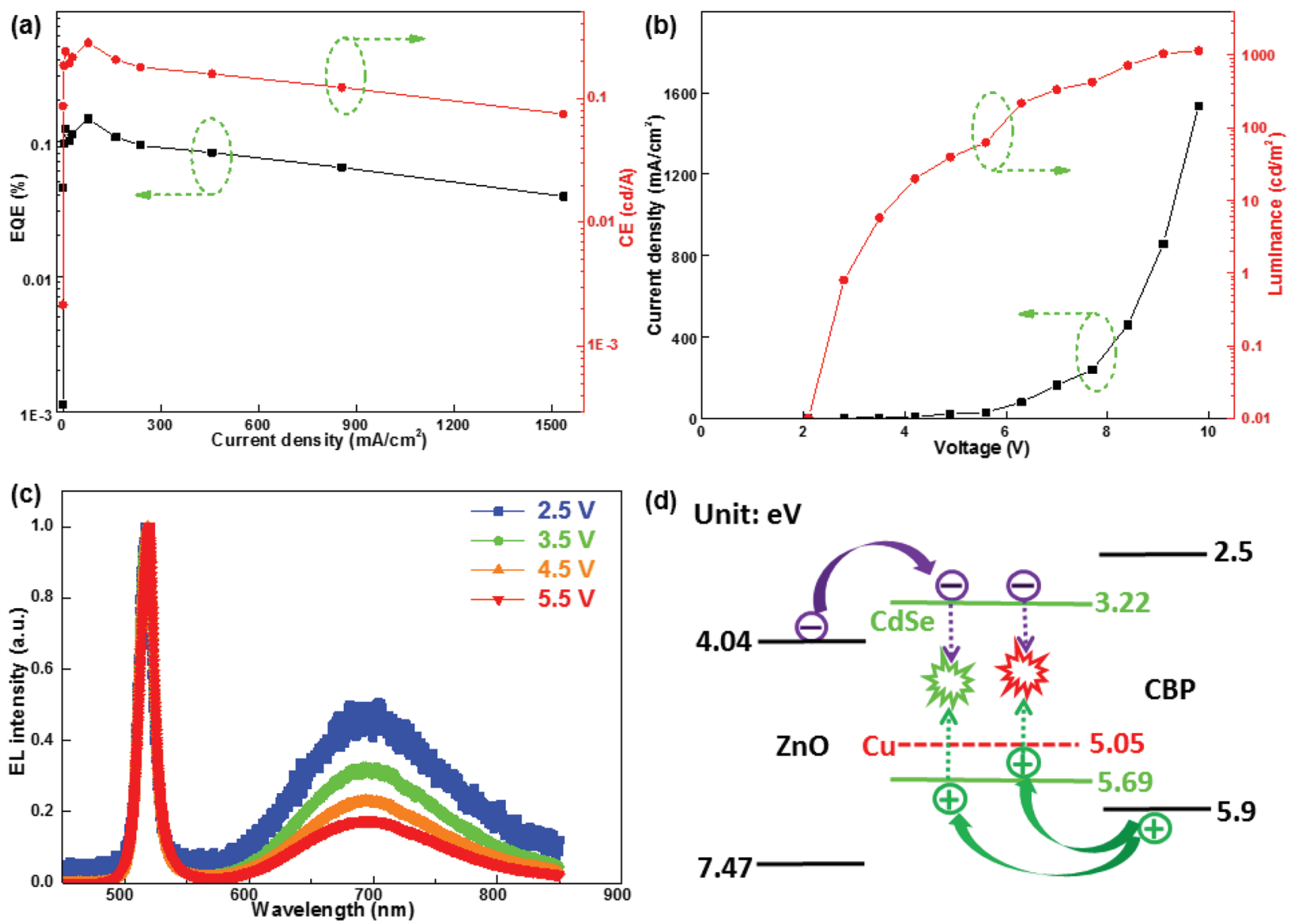

(d)
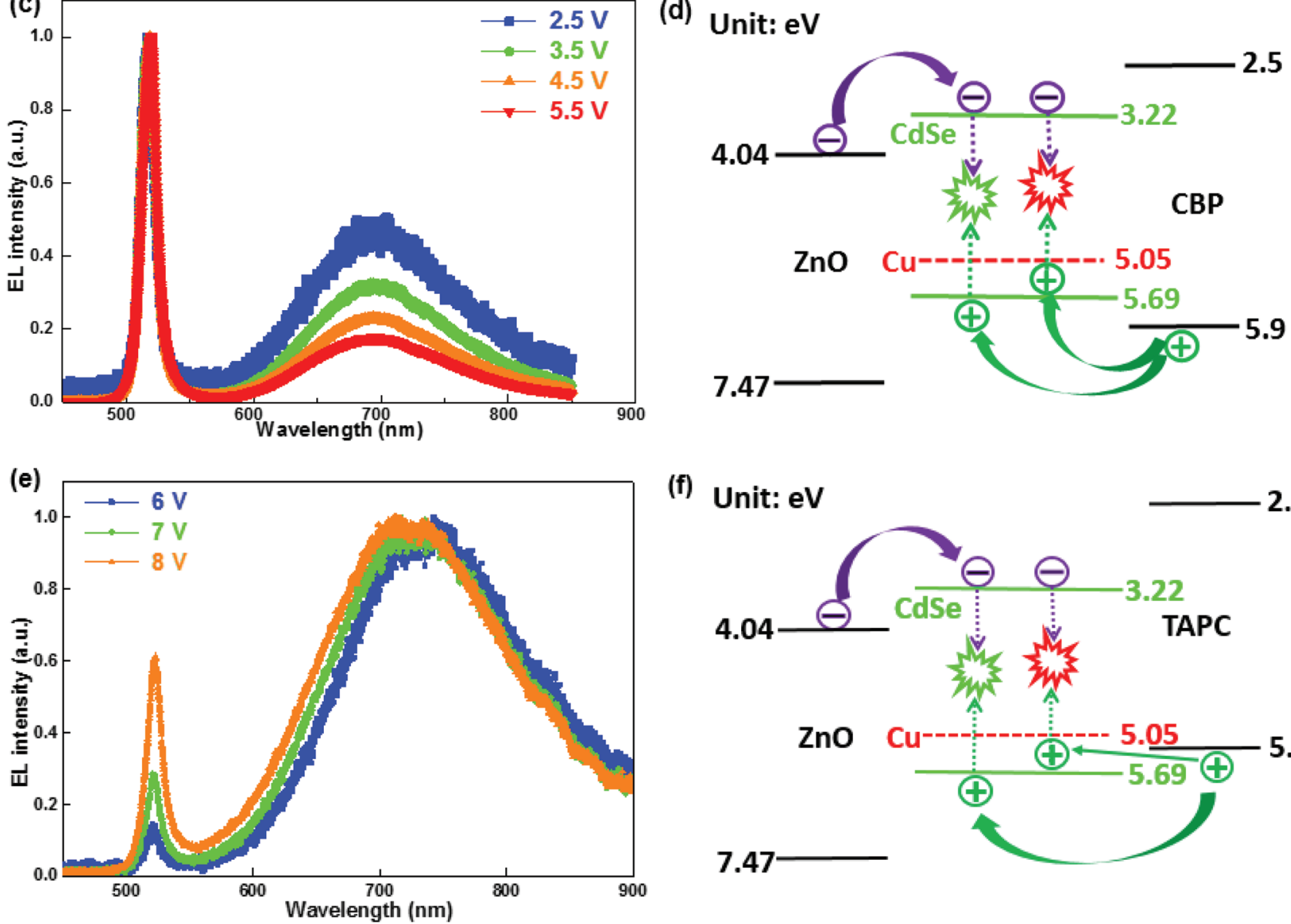

(f)

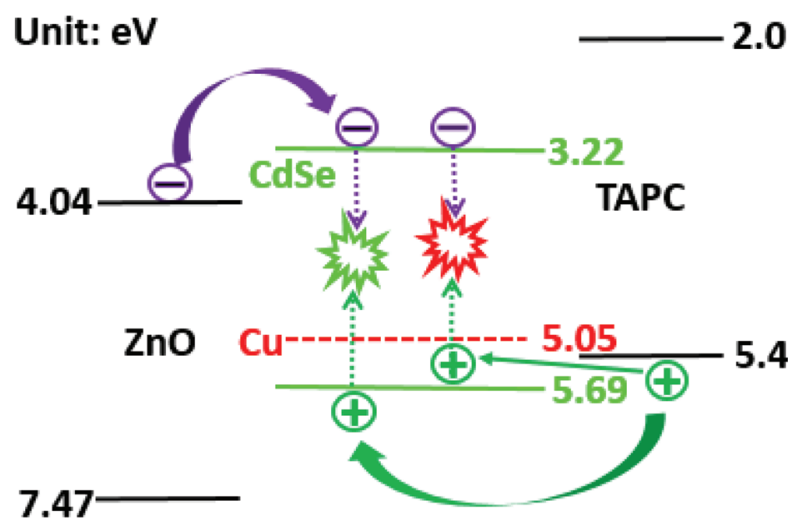

Figure 3. a) EQE and CE of Device B. b) Current density and luminance of Device B. c) EL spectra of Device B at various voltages. d) Schematic of the emission mechanism of Device B. The unit of energy is eV. e) EL spectra of Device $C$ at various voltages. f) Schematic of the emission mechanism of Device C. The unit of energy is eV.

of our Cu-doped NPLs, simultaneous appearance of excitonic and dopant emission (i.e., dual emission) in the EL spectrum suggests further investigations are needed for understanding their origin. To this end, an LED using $60 \mathrm{~nm}$ 1-bis[4-[N,Ndi(4-tolyl)amino]phenyl]-cyclohexane (TAPC) as the HTL was developed (Device C), where the architecture of Device $\mathrm{C}$ is similar to that of Device B except for the HTL. As shown in Figure $3 \mathrm{e}$, the EL emission peak of CdSe (excitonic emission) is lower than that of $\mathrm{Cu}^{+}$in Device $\mathrm{C}$, which is unlike Device B since the EL peak of CdSe is higher than that of $\mathrm{Cu}^{+}$even at the turn-on voltage. In particular, the CdSe excitonic emission is almost prohibited at the turn-on voltage in Device C (Figure S7, 
Supporting Information). Such surprising phenomena suggest that the dual emission from the Cu-doped CQWs can be tuned by deliberately selecting the charge transport layers. Therefore, device engineering is a novel strategy to tune the dual emission of single-nanocrystal LEDs. Since many organic or inorganic charge transport materials exist, using our strategy to tune the dual emissions is very feasible. Thus, apart from the use of an "electrical field" and "material design," "device engineering" may show unique merits for tunable emissions.

The surprising phenomena can be explained as follows. As shown in Figure 3f, since the highest occupied molecular orbital (HOMO) of TAPC $(5.4 \mathrm{eV})$ is located between the $\mathrm{Cu}$ level $(5.05 \mathrm{eV})$ and the VBM of CdSe $(5.69 \mathrm{eV}),{ }^{[48]}$ holes transported from TAPC are readily injected into $\mathrm{Cu}$ due to the barrier-free characteristic. However, injecting holes into CdSe is difficult because of the hole barrier between TAPC and CdSe $(0.29 \mathrm{eV})$. Hence, excitons are almost only generated and recombined to produce $\mathrm{Cu}^{+}$emission at a low electrical field. Moreover, even at a strong electrical field, the CdSe emission is not very prominent compared with the $\mathrm{Cu}^{+}$emission due to the existing hole barrier. These facts imply that the dual emission can be tuned via device engineering, providing deep insight into the emission mechanism of single-nanocrystal LEDs.

To reveal the effect of a high impurity doping concentration in LEDs, CdSe CQWs with a $2.4 \% \mathrm{Cu}$ doping concentration were used as the emitter to fabricate an LED (Device D), where the architecture of Device D is the same as that of Device A except for the EML. As shown in Figure S8 (Supporting Information), dual emission can be produced at different voltages. Moreover, compared with the PL spectra, the EL emission is redshifted, with peaks at $\approx 520$ and $\approx 716 \mathrm{~nm}$ originating from $\mathrm{CdSe}$ and high doping $\mathrm{Cu}^{+}$ions, respectively. In particular, the redshift of the $\mathrm{Cu}$ EL peak in Device D is more obvious than that in Device $\mathrm{B}$, as higher $\mathrm{Cu}$ doping concentrations can lower the CBM of CdSe due to orbital hybridization. ${ }^{[49,50]}$ In addition, a stronger $\mathrm{Cu}^{+}$emission can be observed in Device D. However, despite the higher doping concentration, the emission mechanism of Device D is similar to that of Device B (i.e., Cu functions as a hole trapping center). This fact is further confirmed by the EL spectra of Device D, in which the Cu emission decreases with increasing voltage.

Furthermore, with the encouragement of dual emission from Cu-doped CQWs, we expected that white emission could be generated by combining the $2.4 \% \mathrm{Cu}$-doped CdSe CQWs with a blue emitter. Therefore, by selecting $\mathrm{ZnCdS} / \mathrm{ZnS}$ core/shell CQDs as the blue emitter, an LED was developed (Device E), where the architecture of Device $E$ is the same as that of Device A except for the EML with the mixing ratio of CQD:CQWs = 5:1 (in wt\%). ZnCdS/ZnS core/shell CQDs were synthesized by following an earlier reported procedure, ${ }^{[11]}$ and the absorption and PL spectra of ZnCdS/ZnS are depicted in Figure S9 (Supporting Information), with a PL peak at $475 \mathrm{~nm}$. As shown in Figure $4 \mathrm{a}-\mathrm{c}$, the maximum EQE, CE, PE, and luminance of Device E are $0.029 \%, 0.025 \mathrm{~cd} \mathrm{~A}^{-1}, 0.020 \mathrm{~lm} \mathrm{~W}^{-1}$, and $148 \mathrm{~cd}$ $\mathrm{m}^{-2}$, respectively. In addition, a turn-on voltage of $2.3 \mathrm{~V}$ is obtained, which is among the lowest nanocrystal WLEDs. Remarkably, Device E exhibits white emission, where the blue emission with a peak at $480 \mathrm{~nm}$ originates from $\mathrm{ZnCdS} / \mathrm{ZnS}$ CQDs, and the green/red emissions result from the Cu-doped
CQWs (Figure 4d). To the best of our knowledge, this is the first WLED based on CQWs. Impressively, a maximum color rendering index (CRI) of 62 is obtained, which is the highest reported for WLEDs using two Cd-based nanocrystals. According to Gnade et al.'s report, ${ }^{[52]}$ a CRI $>60$ is acceptable for applications. Additionally, $\geq 3$ nanocrystals are usually required for high CRIs, which inevitably increases the complexity of WLEDs. ${ }^{[53]}$ Although WLEDs using two nanocrystals are more simplified and desirable for low-cost commercialization, their CRI is generally low (e.g., CRI = 14.7 for Bae's WLED with two CQDS ${ }^{[54]}$ ). Therefore, our results provide a novel avenue toward simplified but high-CRI WLEDs.

To further understand the WLED based on CQWs, the exciton decays in Device E were analyzed. Device E possesses a hybrid architecture since $\mathrm{ZnO}$ and $\mathrm{CBP}$ are inorganic and organic charge transport layers, respectively. ${ }^{[36]}$ In addition, since the amount of $\mathrm{ZnCdS} / \mathrm{ZnS}$ CQDs is much higher than that of CQWs, the charge injection is greatly affected by $\mathrm{ZnCdS} / \mathrm{ZnS}$. Moreover, because the electron barrier between the CBMs of $\mathrm{ZnO}$ and $\mathrm{ZnCdS} / \mathrm{ZnS}(3.6 \mathrm{eV})$ is lower than the hole barrier between the HOMO of CBP and the VBM of $\mathrm{ZnCdS} / \mathrm{ZnS}(6.4 \mathrm{eV}),{ }^{[55]}$ the electron injection from $\mathrm{ZnO}$ to $\mathrm{ZnCdS} / \mathrm{ZnS}$ is more effective than the hole injection from $\mathrm{CBP}$ to $\mathrm{ZnCdS} / \mathrm{ZnS}$. As a result, charge imbalance occurs by further considering that the electron mobility of $\mathrm{ZnO}$ is higher than the hole mobility of CBP. This leads to nonradiative Auger recombination, which is detrimental to the efficiency. ${ }^{[36]}$ For the Cu-doped CdSe CQWs, the CdSe and $\mathrm{Cu}$ levels are lower than the HOMO of CBP and the VBM of ZnCdS/ZnS, which is favorable for hole injection. With enhanced electric field, populated holes in CQWs can hop into ZnCdS/ZnS, mitigating the charge imbalance. ${ }^{[53]}$ Hence, the CQWs can contribute to suppressing the Auger decay and enhancing the radiative exciton recombination.

Since two emitters have been mixed in Device E, excitons can be transferred from large-bandgap emitters to small-bandgap emitters via the Förster procedure. ${ }^{[53]}$ Thus, the amount of ZnCdS/ZnS CQDs is fivefold higher than that of CQWs to guarantee white emission because the high-energy excitons from $\mathrm{ZnCdS} / \mathrm{ZnS}$ are readily transferred to the CQWs. To verify this Förster resonant energy transfer (FRET) process, the absorption spectrum of the CQWs and the PL spectrum of the $\mathrm{ZnCdS} /$ ZnS CQDs were compared. ${ }^{[56]}$ As shown in Figure S10 (Supporting Information), a large overlap exists between these two spectra, indicating the efficient FRET in Device E. ${ }^{[53]}$ However, our $\mathrm{ZnCdS} / \mathrm{ZnS}$ is not sufficiently efficient, which limits the efficiency of Device E. To provide evidence for this, a blue LED based on ZnCdS/ZnS CQDs was fabricated (Device F), where the architecture of Device $F$ is the same as that of Device E except for the EML of $\mathrm{ZnCdS} / \mathrm{ZnS}$. As shown in Figure S11 (Supporting Information), the maximum EQE of Device $F$ is only $0.209 \%$. Therefore, to further enhance the efficiency of WLEDs based on CQWs, a facile method is the introduction of highly efficient blue emitters. In addition, by tuning the ratio of CQWs and $\mathrm{ZnCdS} / \mathrm{ZnS}$ CQDs, other white emissions can be obtained, indicating a flexible scheme to achieve WLEDs. For example, a cooler white emission with the CIE coordinates of $(0.22,0.25)$ is produced in Device G (Figure S12, Supporting Information), where the architecture of Device $\mathrm{G}$ is the same as that of Device $\mathrm{E}$ 

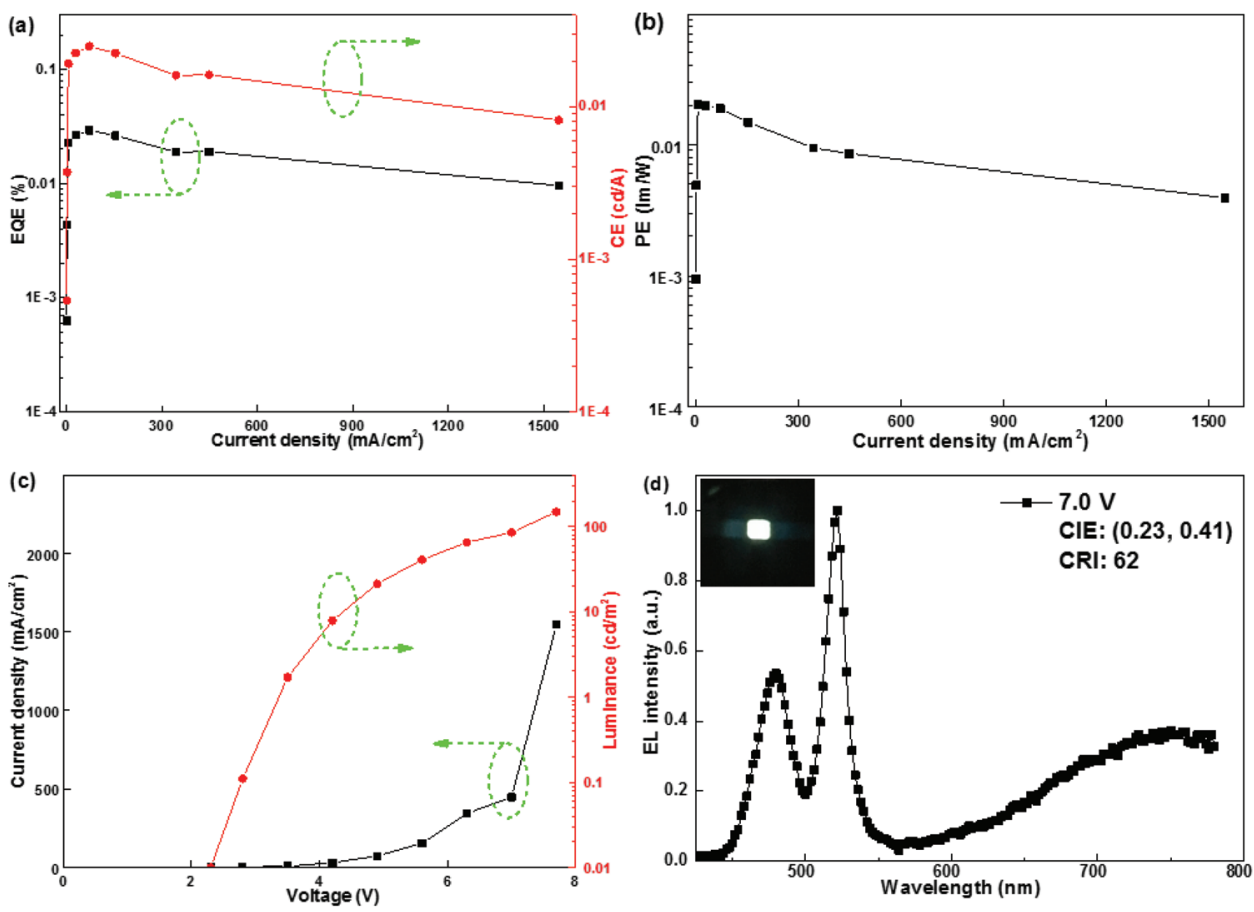

Figure 4. The EL characteristics of Device E. a) EQE and CE, b) power efficiency (PE), c) current density and luminance, and d) EL spectrum. Inset: photograph of Device E under bias.

except for the EML with the ratio of CQDs:CQWs = 10:1. The findings imply that the CQWs can directly operate with the CQDs as the EML and that white emissions can be facilely furnished by adjusting the ratio of CQWs and CQDs, thus bridging the relation between 2D CQWs and OD CQDs. Furthermore, by using different dopant combinations in CQWs (e.g., Mn-, Agdoped CdSe CQWs), the emission spectrum of the dopant is expected to be tuned into a more visible region, which can enable achievement of further high performance and CRI. Finally, it is worth mentioning that the operation lifetime of CQW-LEDs has never been reported, which may be attributed to the instability of CQW-LEDs. The lifetime of our devices made from core-only doped-CQWs is smaller (e.g., $270 \mathrm{~s}$ at $100 \mathrm{~cd} \mathrm{~m}^{-2}$ ). This is comparable to similar undoped CQWs and is only a little longer than some perovskite LEDs (e.g., $80 \mathrm{~s}$ ). ${ }^{[57]}$ However, with CQW heterostructures (e.g., core/shell, core/crown) synthesized by colloidal atomic layer deposition or hot injection methods, LEDs from these doped-CQWs are expected to show high operational lifetime, which are under investigation.

To conclude, LEDs of Cu-doped CQWs have been successfully developed. For the $0 \% \mathrm{Cu}$-doped concentration, the color gamut of the LED can cover 104\% of the Rec. 2020 standard in the CIE 1931 color space. Significantly, the EL FWHM is as narrow as $12 \mathrm{~nm}$. With the $0.5 \% \mathrm{Cu}$-doped concentration, a CQW-LED with dual emission has been reported. Impressively, the dual emission can be tuned via device engineering, which is an unreported mechanism for single-nanocrystal LEDs. Moreover, with the 2.4\% Cu-doped concentration, the WLED based on CQWs has been demonstrated. Such results undrape new advantages of CQW-LEDs, begin the step that LEDs based on CQWs can possess polychromatic emission apart from previous discovered monochromatic emission, and open a new avenue to realize LEDs by employing 2D impurity-doped CQWs that can be further extended to the use of other impurities (e.g., Mn, Ag).

\section{Supporting Information}

Supporting Information is available from the Wiley Online Library or from the author.

\section{Acknowledgements}

B.L. and M.S. contributed equally to this work. The authors declare no competing financial interest. This research is supported by the National Research Foundation, Prime Minister's Office, Singapore under its Investigatorship program (NRF-NRFI2016-08) and the Singapore Agency for Science, Technology and Research (A*STAR) SERC Pharos Program under grant no. 15273 00025. H.V.D. gratefully acknowledges TUBA. The authors thank ACS ChemWorx Authoring Services (https:// es.acschemworx.acs.org/en/) for providing linguistic assistance during the preparation of this manuscript.

\section{Conflict of Interest}

The authors declare no conflict of interest.

\section{Keywords}

colloidal quantum wells, dual emission, impurity doping, light-emitting diodes, nanoplatelets

Received: April 20, 2019

Revised: June 28, 2019

Published online: August 5, 2019 
[1] S. Ithurria, M. D. Tessier, B. Mahler, R. P. S. M. Lobo, B. Dubertret, A. L. Efros, Nat. Mater. 2011, 10, 936.

[2] J. Q. Grim, S. Christodoulou, F. Di Stasio, R. Krahne, R. Cingolani, L. Manna, I. Moreels, Nat. Nanotechnol. 2014, 9, 891.

[3] C. E. Rowland, I. Fedin, H. Zhang, S. K. Gray, A. O. Govorov, D. V. Talapin, R. D. Schaller, Nat. Mater. 2015, 14, 484.

[4] A. Riedinger, F. D. Ott, A. Mule, S. Mazzotti, P. N. Knusel, S. J. P. Kress, F. Prins, S. C. Erwin, D. J. Norris, Nat. Mater. 2017, 16, 743 .

[5] D. V. Talapin, J.-S. Lee, M. V. Kovalenko, E. V. Shevchenko, Chem. Rev. 2010, 110, 389.

[6] M. D. Tessier, C. Javaux, I. Maksimovic, V. Loriette, B. Dubertret, ACS Nano 2012, 6, 6751.

[7] B. Mahler, B. Nadal, C. Bouet, G. Patriarche, B. Dubertret, J. Am. Chem. Soc. 2012, 134, 18591.

[8] M. D. Tessier, P. Spinicelli, D. Dupont, G. Patriarche, S. Ithurria, B. Dubertret, Nano Lett. 2014, 14, 207.

[9] L. T. Kunneman, J. M. Schins, S. Pedetti, H. Heuclin, F. C. Grozema, A. J. Houtepen, B. Dubertret, L. D. A. Siebbeles, Nano Lett. 2014, 14, 7039.

[10] C. She, I. Fedin, D. S. Dolzhnikov, P. D. Dahlberg, G. S. Engel, R. D. Schaller, D. V. Talapin, ACS Nano 2015, 9, 9475.

[11] F. T. Rabouw, J. C. Bok, P. Spinicelli, B. Mahler, M. Nasilowski, S. Pedetti, B. Dubertret, D. Vanmaekelbergh, Nano Lett. 2016, 16, 2047.

[12] L. Jing, S. V. Kershaw, Y. Li, X. Huang, Y. Li, A. L. Rogach, M. Gao, Chem. Rev. 2016, 116. 10623.

[13] Y. Ling, Z. Yuan, Y. Tian, X. Wang, J. C. Wang, Y. Xin, K. Hanson, B. Ma, H. Gao, Adv. Mater. 2016, 28, 305.

[14] C. Qin, T. Matsushima, A. S. Sandanayaka, Y. Tsuchiya, C. Adachi, J. Phys. Chem. Lett. 2017, 8, 5415.

[15] Z. Chen, B. Nadal, B. Mahler, H. Aubin, B. Dubertret, Adv. Funct. Mater. 2014, 24, 295.

[16] V. L. Colvin, M. C. Schlamp, A. P. Alivisatos, Nature 1994, 370, 354.

[17] Y. X. Yang, Y. Zheng, W. R. Cao, A. Titov, J. Hyvonen, J. R. Manders, J. G. Xue, P. H. Holloway, L. Qian, Nat. Photonics 2015, 9, 259.

[18] X. L. Dai, Z. X. Zhang, Y. Z. Jin, Y. Niu, H. J. Cao, X. Y. Liang, L. W. Chen, J. P. Wang, X. G. Peng, Nature 2014, 515, 96.

[19] B. Liu, L. Wang, D. Y. Gao, J. H. Zou, H. L. Ning, J. B. Peng, Y. Cao, Light: Sci. Appl. 2016, 5, e16137.

[20] Z. Shi, S. Li, Y. Li, H. Ji, X. Li, D. Wu, T. Xu, Y. Chen, Y. Tian, Y. Zhang, C. Shan, G. Du, ACS Nano 2018, 12, 1462.

[21] F. Fan, P. Kanjanaboos, M. Saravanapavanantham, E. Beauregard, G. Ingram, E. Yassitepe, M. M. Adachi, O. Voznyy, A. K. Johnston, G. Walters, G. Kim, Z.-H. Lu, E. H. Sargent, Nano Lett. 2015, 15, 4611.

[22] B. Liu, S. Delikanli, Y. Gao, D. Dede, K. Gungor, H. V. Demir, Nano Energy 2018, 47, 115

[23] U. Giovanella, M. Pasini, M. Lorenzon, F. Galeotti, C. Lucchi, F. Meinardi, S. Luzzati, B. Dubertret, S. Brovelli, Nano Lett. 2018, 18,3441

[24] R. Zhu, Z. Luo, H. Chen, Y. Dong, S.-T. Wu, Opt. Express 2015, 23, 23680.

[25] S. Kumar, J. Jagielski, N. Kallikounis, Y.-H. Kim, C. Wolf, F. Jenny, T. Tian, C. J. Hofer, Y.-C. Chiu, W. J. Stark, T.-W. Lee, C.-J. Shih, Nano Lett. 2017, 17, 5277.

[26] Y. Tong, E.-P. Yao, A. Manzi, E. Bladt, K. Wang, M. Doblinger, S. Bals, P. Muller-Buschbaum, A. S. Urban, L. Polavarapu, Adv. Mater. 2018, 30, 1801117.

[27] M. Lorenzon, V. Pinchetti, F. Bruni, W. K. Bae, F. Meinardi, V. I. Klimov, S. Brovelli, Nano Lett. 2017, 17, 1071.

[28] V. Pinchetti, F. Meinardi, A. Camellini, G. Sirigu, S. Christodoulou, W. K. Bae, F. D. De Donato, L. Manna, M. Zavelani-Rossi, I. Moreels, V. I. Klimov, S. Brovelli, ACS Nano 2016, 10, 6877.
[29] Q. Lin, N. S. Makarrov, W.-K. Koh, K. A. Velizhanin, C. M. Cirloganu, H. Luo, V. I. Klimov, J. M. Pietryga, ACS Nano 2015, 9, 539.

[30] S. Brovelli, W. K. Bae, C. Galland, U. Giovanella, F. Meinardi, V. I. Klimov, Nano Lett. 2014, 14, 486.

[31] R. Xie, X. Peng, J. Am. Chem. Soc. 2009, 131, 10645.

[32] V. A. Vlaskin, N. Janssen, J. V. Rijssel, R. Beaulac, D. R. Gamelin, Nano Lett. 2010, 10, 3670.

[33] A. H. Khan, A. Dalui, S. Mukherjee, C. U. Segre, D. D. Sarma, S. Acharya, Angew. Chem., Int. Ed. 2015, 54, 2643.

[34] K. E. Knowles, K. H. Hartstein, T. B. Kilburn, A. Marchioro, H. D. Nelson, P. J. Whitham, D. R. Gamelin, Chem. Rev. 2016, 116, 10820.

[35] M. Sharma, K. Gungor, A. Yeltik, M. Olutas, B. Guzelturk, Y. Kelestemur, T. Erdem, S. Delikanli, J. R. McBride, H. V. Demir, Adv. Mater. 2017, 29, 1700821.

[36] B. S. Mashford, M. Stevenson, Z. Popovic, C. Hamilton, Z. Zhou, C. Breen, J. Steckel, V. Bulovic, M. Bawendi, S. Coe-Sullivan, P. T. Kazlas, Nat. Photonics 2013, 7, 407.

[37] B. Liu, L. Wang, H. Gu, H. Sun, H. V. Demir, Adv. Opt. Mater. 2018, 6, 1800220.

[38] D. Liang, Y. Peng, Y. Fu, M. J. Shearer, J. Zhang, J. Zhai, Y. Zhang, R. J. Hamers, T. L. Andrew, S. Jin, ACS Nano 2016, 10, 6897.

[39] T. Zhao, H. Liu, M. E. Ziffer, A. Rajagopal, L. Zuo, D. S. Ginger, X. Li, A. K. Y. Jen, ACS Energy Lett. 2018, 3, 1662.

[40] S. N. Inamdar, P. P. Ingole, S. K. Haram, ChemPhysChem 2008, 9, 2574.

[41] S. Maiti, J. Dana, Y. Jadhav, T. Debnath, S. K. Haram, H. N. Ghosh, J. Phys. Chem. C 2017, 121, 27233.

[42] J. Kwak, W. K. Bae, D. Lee, I. Park, J. Lim, M. Park, H. Cho, H. Woo, D. Y. Yoon, K. Char, S. Lee, C. Lee, Nano Lett. 2012, 12, 2362.

[43] J.-R. Gong, L.-J. Wan, S.-B. Lei, C.-L. Bai, X.-H. Zhang, S.-T. Lee, J. Phys. Chem. B 2005, 109, 1675.

[44] B. Liu, H. Nie, X. Zhou, S. Hu, D. Luo, D. Gao, J. Zou, M. Xu, L. Wang, Z. Zhao, A. Qin, J. Peng, H. Ning, Y. Cao, B. Z. Tang, Adv. Funct. Mater. 2016, 26, 776.

[45] L. P. Lu, D. Kabra, R. H. Friend, Adv. Funct. Mater. 2012, 22, 4165.

[46] G. A. H. Wetzelaer, M. Kuik, H. T. Nicolai, P. W. M. Blom, Phys. Rev. B 2011, 83, 165204

[47] N. K. Kumawat, N. Jain, A. Dey, K. L. Narasimhan, D. Kabra, Adv. Funct. Mater. 2017, 27, 1603219.

[48] X. K. Liu, Z. Chen, C. J. Zheng, M. Chen, W. Liu, X. H. Zhang, C. S. Lee, Adv. Mater. 2015, 27, 2025.

[49] J. T. Wright, K. Forsythe, J. Hutchins, R. W. Meulenberg, Nanoscale 2016, 8, 9417.

[50] M. Sharma, M. Olutas, A. Yeltik, Y. Kelestemur, A. Sharma, S. Delikanli, B. Guzelturk, K. Gungor, J. R. McBride, H. V. Demir, Chem. Mater. 2018, 30, 3265.

[51] Y. Wang, K. S. Leck, V. D. Ta, R. Chen, V. Nalla, Y. Gao, T. He, H. V. Demir, H. Sun, Adv. Mater. 2015, 27, 169.

[52] U. S. Bhansali, H. Jia, I. W. Oswald, M. A. Omary, B. E. Gnade, Appl. Phys. Lett. 2012, 100, 183305.

[53] K.-H. Lee, C. Han, H. Kang, H. Ko, C. Lee, J. Lee, N. Myoung, S.-Y. Yim, H. Yang, ACS Nano 2015, 9, 10941.

[54] W. K. Bae, J. Lim, D. Lee, M. Park, H. Lee, J. Kwak, K. Char, C. Lee, S. Lee, Adv. Mater. 2014, 26, 6387.

[55] T. Cheng, Z. Wang, S. Jin, F. Wang, Y. Bai, H. Feng, B. You, Y. Li, T. Hayat, Z. Tan, Adv. Opt. Mater. 2017, 5, 1700035.

[56] G. J. Supran, Y. Shirasaki, K. W. Song, J.-M. Caruge, P. T. Kazlas, S. Coe-Sullivan, T. L. Andrew, M. G. Bawendi, V. Bulovic, MRS Bull. 2013, 38, 703

[57] X. Zhang, H. Liu, W. Wang, J. Zhang, B. Xu, K. L. Karen, Y. Zheng, S. Liu, S. Chen, K. Wang, X. W. Sun, Adv. Mater. 2017, 29, 1606405. 